Tropical Journal of Pharmaceutical Research April 2021; 20 (4): 709-714

ISSN: $1596-5996$ (print); 1596-9827 (electronic)

(C) Pharmacotherapy Group, Faculty of Pharmacy, University of Benin, Benin City, 300001 Nigeria.

Available online at http://www.tjpr.org

Original Research Article

http://dx.doi.org/10.4314/tjpr.v20i4.7

\title{
Potential regulatory effects of CXCR2 and CPEB1 on cardiac cachexia
}

\author{
Yang Li ${ }^{1}$, Congyu Zhang ${ }^{2}$, Qian Tong ${ }^{1}$, Xiaoxing Feng ${ }^{1 *}$ \\ ${ }^{1}$ Department of Cardiovascular Medicine, The First Hospital of Jilin University, ${ }^{2}$ Department of Cardiovascular Medicine, \\ Affiliated Hospital of Changchun University of Traditional Chinese Medicine, Changchun, Jilin, 130021, China \\ *For correspondence: Email: fengxx@jlu.edu.cn; Tel: 00860-0431-88782207
}

Sent for review: 22 July 2020

Revised accepted: 17 March 2021

\begin{abstract}
Purpose: To investigate the effect of $C-X-C$ motif chemokine receptor 2 (CXCR2) and cytoplasmic polyadenylation element binding protein 1 (CPEB1) which are linked to inflammatory and cellular senescence, on cardiac cachexia.

Methods: Early and advanced stages of cardiac cachexia were established in mice using exogenous injection of single doses of growth differentiation factor 11 (GDF11, 3.0 and $6.0 \mathrm{mg} / \mathrm{kg}$, respectively). The expressions of CXCR2 and CPEB1 were assayed using immonohistochemistry and Western blotting.

Results: Following two weeks of GDF11 injection, cardiac tissue mass was reduced by 23 and $39 \%$ in early and advanced stages cardiac cachexia, respectively, in mice. Histology showed progressive loss of cardiac muscle and cardiomyocyte shrinkage in early stage, with extensive cardiac muscle wasting and disruption of cardiac arche structure in the advanced stage. Immunohistochemistry and Western blotting showed that CXCR2 was 4.6 times overexpressed in the initial stage of cachexia, but was restricted to only 2.4-fold overexpression in advanced stage of cardiac cachexia. The results showed that CXCR2 and its higher expression in early stages of cardiac cachexia prepared the cardiac microenvironment for vast changes during which its expression was limited. There were 2.2-fold and 5.5-fold higher expressions of CPEB1 in early and advanced stages of cardiac cachexia, respectively, indicating its role in cardiac muscle wasting and remodelling in cellular senescence.
\end{abstract}

Conclusion: CXCR2 overexpression modulates extensive cardiac muscle loss in cardiac cachexia, thus affording a strategy for the management of this pathological condition.

Keywords: Cardiac cachexia, Growth differentiation factor 11 (GDF11), C-X-C motif chemokine receptor 2 (CXCR2), Cytoplasmic polyadenylation element binding protein 1 (CPEB1), Muscle wasting

This is an Open Access article that uses a fund-ing model which does not charge readers or their institutions for access and distributed under the terms of the Creative Commons Attribution License (http://creativecommons.org/licenses/by/4.0) and the Budapest Open Access Initiative (http://www.budapestopenaccessinitiative.org/read), which permit unrestricted use, distribution, and reproduction in any medium, provided the original work is properly credited.

Tropical Journal of Pharmaceutical Research is indexed by Science Citation Index (SciSearch), Scopus, International Pharmaceutical Abstract, Chemical Abstracts, Embase, Index Copernicus, EBSCO, African Index Medicus, JournalSeek, Journal Citation Reports/Science Edition, Directory of Open Access Journals (DOAJ), African Journal Online, Bioline International, Open-J-Gate and Pharmacy Abstracts

\section{INTRODUCTION}

Cardiac cachexia is a condition of muscle loss due to chronic inflammation, insulin resistance and functional incapability as a result of elevated neurohormonal activation [1]. At the clinical level, it is widely unrecognized in the early stages, leading to poor quality of life and ultimately premature death [2]. In 2014, Europe alone had 1.2 million individual cardiac cachexia cases, with a mortality of over 20 - $40 \%$ [3]. Cardiac cachexia and its associated complex 
pathophysiology have been studied to a certain extent, but its prognosis is still not well understood [4]. According to a recent survey, approximately $10 \%$ of heart failure patients have cardiac cachexia conditions [5].

Although recent advances have been made in understanding the mechanisms involved in the etiology of cardiac cachexia, translating them to achieve clinical success is still a challenge. Lack of suitable animal models that mimic the etiology of the disease is also considered as one of the major drawbacks. At present, there is no therapy or treatment for preventing or suppressing cardiac cachexia. Therefore, studies in this aspect are needed to evolve more effective ways of reversing cardiac cachexia, instead of treating the symptoms alone.

It is known that CXCR2 is a chemokine receptor which plays a major role in the migration of neutrophils to an inflammatory site [6]. Moreover, CXCR3 is significantly involved in metastasis, angiogenesis and melanoma growth [7]. There are also reports available in relation to pancreatic cancer and atrophy in which CXCR2 is the major causative agent [8]. Cytoplasmic polyadenylation element binding protein 1 (CPEB1) binds to RNA in a sequence-specific manner, and it regulates the length of the poly (A) tail of mRNA, which in turn represses or promotes the translation of its targets [9]. The epigenetic regulation of CPEB1 and its overexpression in glioma are involved in cellular senescence [10]. Not much in known about the effects of CXCR2 and CPEB1 in cardiac cachexia. In the present study, the effects of CXCR2 and CPEB1 on cachexia, and the mechanism involved were investigated using a cardiac cachexia mouse model.

\section{EXPERIMENTAL}

\section{Mouse model of cachexia}

Thirteen-week-old male C57BL/6 mice $(n=36)$ were purchased from Jackson Laboratory, Shanghai, China. Following initial examination, the animals were kept in the laboratory cage for 1 week to acclimatize. Before using the animals for experiments, prior approval for animal handling and protocol were granted by The First Hospital of Jilin University ethical committee (approval no. FHJU031812). The mice were allowed ad libitum access to water and chow, and maintained under 12-h light/12-h dark cycle at a humidity of $55 \%$. Cardiac cachexia was induced using growth differentiation factor 11 (GDF11) in line with a protocol described earlier [11]. Following acclimatization, the first group of mice $(n=12)$ received a single dose of GDF11 $(3.0 \mathrm{mg} / \mathrm{kg})$ along with a vehicle $(60 \mathrm{mM}$ sodium acetate buffer, pH 5.0 and $10 \%$ Trehelose) via intraperitoneal injection. Similarly, a second group of mice $(n=12)$ received a single dose of GDF11 $(6.0 \mathrm{mg} / \mathrm{kg})$ along with vehicle, while the third group ( $\mathrm{n}=12)$ received vehicle only, without GDF11 injection. After two weeks, the mice were euthanized with cervical dislocation, followed by cardiac puncture. Then, the cardiac tissues were dissected out.

\section{Histology and immunohistochemistry}

Following excision of mice hearts, the tissues were washed with $1 \mathrm{X}$ PBS and immediately fixed in $10 \%$ formaldehyde. After $48 \mathrm{~h}$ of fixation, the tissues are washed with water and dehydrated in gradient concentrations of isopropyl alcohol. This was followed with tissue clearing in xylene, embedding in wax and sectioning into $6-\mu \mathrm{m}$ slices. The sections were placed on a slide, processed further and finally stained using haematoxylin and eosin (H\&E). For immunohistochemistry, the deparaffinized slides were subjected to antigen retrieval in a boiling solution of $0.01 \mathrm{M}$ sodium citrate buffer ( $\mathrm{pH} \mathrm{6.0)}$ for $20 \mathrm{~min}$. The slides were then rinsed with 1X TBST buffer and blocked with $4 \%$ BSA (in TBST) for $40 \mathrm{~min}$ at room temperature. Thereafter, the slides were incubated with diluted antibodies, i.e, anti-CXCR2 (Abcam ab14935; 1:300 dilution) and antiCPEB1 (Abcam - ab3465; $1: 500$ dilution) at $4{ }^{\circ} \mathrm{C}$ for $6 \mathrm{~h}$. Then, the slides were washed 3 times with $1 \mathrm{X}$ TBST buffer to remove unbound antibodies, and further incubated with HRP-conjugated secondary antibody at room temperature for $2 \mathrm{~h}$. Following the washing step, the slides were incubated with DAB solution for $20 \mathrm{~min}$ in a dark environment to form brown colour signals.

\section{Western blotting}

Each mouse heart tissue was homogenised in ice cold condition with RIPA buffer and the proteins present in the sample were quantified using BCA protein assay kit (Thermo Scientific, Shanghai, China). Equal amounts of proteins were resolved in SDS-polyacrylamide gel electrophoresis at $50 \mathrm{~V}$ for $4 \mathrm{~h}$. The separated proteins were transferred unto PVDF membrane. The membrane was blocked by incubation with 5 $\%$ skimmed milk in 1X TBST, followed by immunoblotting with antibodies (antiCXCR2, Abcam - ab14935; 1:200 dilution) and antiCPEB1 (Abcam - ab3465; 1:300 dilution) overnight at $4{ }^{\circ} \mathrm{C}$. After washing with $1 \mathrm{X}$ TBST, the membrane was incubated with suitable 
secondary antibody and the specific signals were detected using DAB kit.

\section{Statistical analysis}

Results obtained from triplicate experiments are expressed as mean \pm standard deviation (SD) and were analysed using SPSS software version 25.0. Differences were considered statistically significant at $p$ values less than 0.05 .

\section{RESULTS}

\section{Mice developed early and advanced stages of cardiac cachexia}

To assay the expressions of CXCR2 and CPEB1 in different pathological stages of cardiac cachexia disease, a mouse model of early and advanced stage cardiac cachexia was established using exogenous injection of GDF11 at doses of 3.0 and $6.0 \mathrm{mg} / \mathrm{kg}$, respectively, as described. Fourteen days after GDF11 injection, the mice were sacrificed and heart tissue was excised and weighed. In the mice injected with $3.0 \mathrm{mg} / \mathrm{kg}$ of GDF11, the cardiac weight was reduced by $23 \%$ following 14 days of incubation, implying cardiac muscle loss. Similarly, mice injected with GDFII at a dose of $6.0 \mathrm{mg} / \mathrm{kg}$ had cardiac weight loss of up to $39 \%$, indicating the adverse effect of the drug.

The dissected heart tissues from initial and advanced stages of cardiac cachexia mice were compared with heart tissue from control mice. Histological examination of heart tissues from cachexia mice revealed progressive tissue loss and reduction in cardiomyocyte size with advancement in cardiac cachexia (Figures $1 \mathrm{~A}$ C). In control cardiac tissue, the cardiomyocytes were compactly packed and well organized, with large nuclei (Figure $1 \mathrm{~A}$ ). However, in early stage-cardiac cachexia mice, muscle loss associated with shrinkage of cardiomyocytes was evident (Figure $1 \mathrm{~B}$ ). In advanced stage of cardiac cachexia, extensive muscle loss and total disruption of cardiac arche structure were visible (Figure $1 \mathrm{C}$ ).

\section{CXCR2 triggered cardiac cachexia}

In order to measure the expressions of CXCR2 in early and advanced stages of cardiac cachexia, immunohistochemistry was performed using anti-CXCR2 antibody and the data are shown in Figures $2 \mathrm{~A}-\mathrm{C}$. In control cardiac tissue, the expression of CXCR2 was restricted to very minimal levels (Figure 2 A) but its expression was extensively increased in early damages associated with cardiac muscle loss (Figure 2 B). In advanced stages of cardiac cachexia, the intensity of CXCR2 expression was significantly restricted, but its signal was visible throughout the tissue layer (Figure $2 \mathrm{C}$ ).

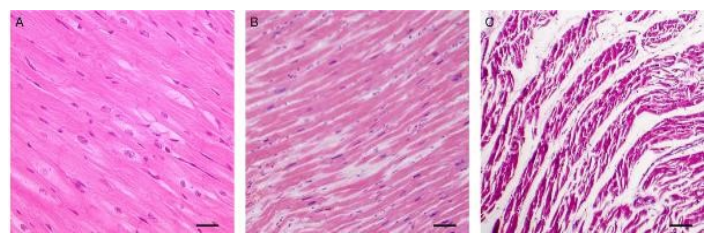

Figure 1: Effect of GDF11 on histomorphology of cardiac tissues of mice with early and advanced stage of cardiac cachexia. A: Histomorphology of control cardiac tissue with compact cellular structure and prominent enlarged nucleus. B: Histomorphology of cardiac tissue mice given GDF11 $(3.0 \mathrm{mg} / \mathrm{kg})$ injection (early cachexia mice) showing slight muscle loss and cardiomyocyte shrinkage. C: Histomorphology of cardiac tissue mice given GDF11 $(6.0 \mathrm{mg} / \mathrm{kg})$ injection (advanced cachexia mice) showing extensive muscle loss and cardiac remodeling

\section{CPEB1 was associated with extensive damage in cardiac cachexia}

In control cardiac tissue, CPEB1 expression was highly restricted (Figure 2 D). However, surprisingly, CPEB1 which is associated with cellular senescence, had restricted expression in early cardiac cachexia (Figure $2 \mathrm{E}$ ). As the damages and cardiac wasting condition progressed, there was significant overexpression of CPEB1 throughout the cardiac tissue layers (Figure $2 \mathrm{~F}$ ).
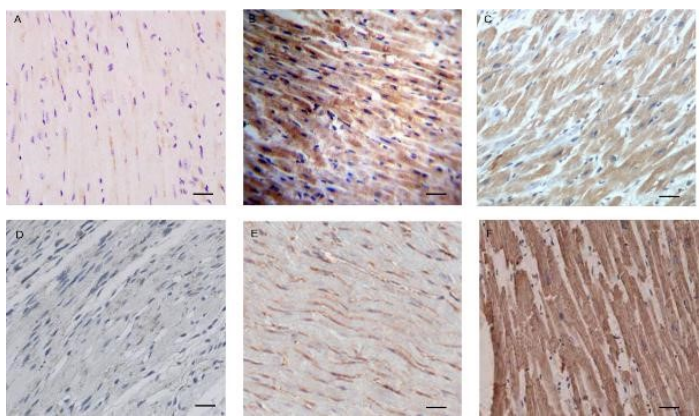

Figure 2: Immunohistochemical analysis of expressions of CXCR2 and CPEB1 in early and advanced cardiac cachexia: A: CXCR2 expression was limited in control cardiac tissue from mice injected with vehicle only. B: Extensive overexpression of CXCR2 in early stages of cardiac cachexia. C: CXCR2 expression was lower at the early stage, but was optimally overexpressed, relative to control tissue. D: Limited CPEB1 signals in control cardiac tissue. E: High expression of CPEB1 in early cardiac cachexia. F: CPEB1 expression extremely increased at the end stage of cardiac cachexia 
Quantitative analysis of expression levels of CXCR2 and CPEB1 using Western blotting

To determine the expression levels of CXCR2 and CPEB, and to confirm the results obtained using immunohistochemistry, western blotting was performed using anti-CXCR2 and antiCPEB1 antibodies. The results (Figure 3 and Figure 4) correlated with the immunohistochemistry data in which CXCR2 expression was 4.6-fold higher in the early-stage cardiac cachexia than in the control cardiac tissue. In contrast, CXCR2 expression in advanced stage cardiac cachexia condition was only 2.4-fold higher than that in control tissue. Similarly, the results for CPEB1 were consistent with the immunohistochemistry data. In early cardiac cachexia, CPEB1 showed 2.2 times higher expression than that in control tissue, and 5.5 times higher expression than the corresponding expression in control tissue in advanced stage cardiac cachexia (Figure 3 and Figure 4). The results were normalized to $\beta$ actin.

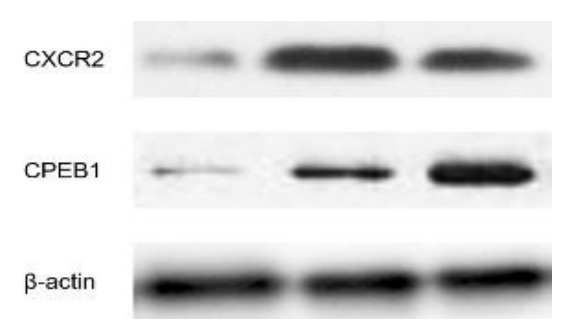

Figure 3: Expressions of CXCR2 and CPEB1 in cardiac cachexia. Lane 1 shows CXCR2, CPEB1 and $\beta$-actin band signals from control cardiac tissue. Lane 2 shows CXCR2, CPEB1 and $\beta$-actin expression in early-stage cardiac cachexia (Early $\mathrm{CC}$ ). Lane 3 indicates CXCR2, CPEB1 and $\beta$-actin expressions in advanced-stage cardiac cachexia

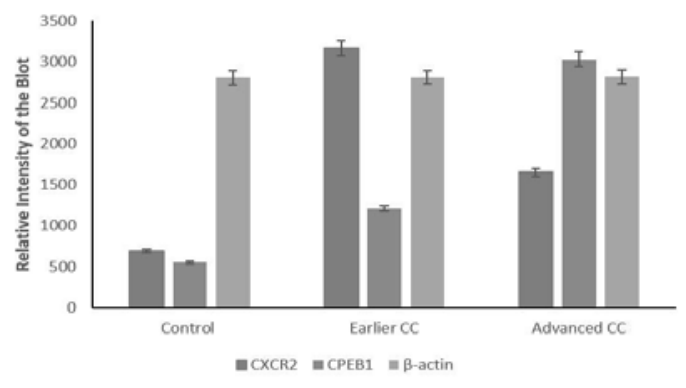

Figure 4: Relative expressions levels of CXCR2 and CPEB1. CXCR2 had 4.6 and 2.4 times higher expressions in early and advanced stages of cardiac cachexia, respectively, than in control cardiac tissue. CPEB1 had 2.2 times higher expression in early cardiac cachexia, but was overexpressed up to 5.5 times in advanced stage of cardiac cachexia. $P<0.05$ was considered as statistically significant. The results were normalized to $\beta$-actin

\section{DISCUSSION}

The use of an appropriate animal model is key to the success of any animal-based research. The use of excess GDF11 for inducing cardiac cachexia is an established model which is similar to the myostatin effect on muscle wasting [12]. Another important feature of using GDF11 is that it does not alter gene expression patterns, and it exactly elucidates cardiac cachexia condition [13]. In this study, the effect of GDF11 was also evident in cardiac muscle loss up to 23 and $39 \%$ in early and advanced stages of cardiac cachexia, respectively. Histological studies showed the development of peculiar features similar to those of cardiac cachexia, with cardiac tissue loss associated with cardiomyocyte shrinkage [14]. The pathological changes associated with early and advanced stages of cardiac cachexia are due to increased inflammatory responses, apoptosis and catabolic processes [15]. The expression of CXCR2 was highly elevated in early stages of cardiac cachexia. This was correlated with previous results on its role in the initiation of neuroinflammation by preparing the cerebral endothelial cells for leukocyte recruitment, indicating its importance in initiating pathological responses [16]. The higher expression of CXCR2 and initiation of cardiac muscle loss in the early stage, and its downregulation at the advanced stage of cardiac cachexia show its important role in initiating basal level changes in early-stage damages. In a recent research, it was shown that IL-8 exerted skeletal muscular atrophy effect via activation of CXCR2 receptor [8]. Interestingly, in this research it was shown that CXCR2 was initially upregulated in early stage, but not in the end stage of cardiac cachexia, which clearly implies its role prior to initiation of high levels of cardiac tissue loss.

In this study, CPEB1 was upregulated to a limited extent in early cardiac cachexia, but it was upregulated in advanced cardiac cachexia. It is important to know that CPEB1 is involved in the regulation of cell cycle, gametogenesis, cellular senescence and inflammation [17]. A comparison of the CPEB1 data in early and advanced stages of cardiac cachexia in correlation with cellular senescence shows its major role in advanced stages due to increased expression which was evident in muscle loss. The optimal CPEB1 overexpression in early stage of cardiac cachexia and higher CXCR2 expression showed that CPEB1 may regulate CXCR2 in early stages for the initiation of pathophysiological changes. The higher level of CPEB1 overexpression may initiate cellular 
senescence which is linked to cardiac muscle loss.

\section{CONCLUSION}

Early and advanced stages of cardiac cachexia in a mouse model have successfully been established using high concentrations of GDF11. The expression of CXCR2 was upregulated in early cardiac cachexia. On the other hand, CPEB1 overexpression was observed in advanced cardiac cachexia. Thus, CXCR2 modulates cardiac tissue at the initial stage of cardiac cachexia which facilitates cellular senescence and muscle loss through upregulated expression of CPEB1 at the advanced stage of cardiac cachexia, and hence providing a potential strategy for the management of the disease.

\section{DECLARATIONS}

\section{Conflict of interest}

No conflict of interest is associated with this work.

\section{Contribution of authors}

We declare that all the authors read the final version of the manuscript and approved it for publication. Yang $\mathrm{Li}$ and Congyu Zhang contributed to this work equally. Yang Li, Congyu Zhang and Xiaoxing Feng conceived and designed the study, Yang Li, Congyu Zhang, Qian Tong, Xiaoxing Feng collected and analysed the data, while Xiaoxing Feng wrote the manuscript. Yang $\mathrm{Li}$ and Congyu Zhang contributed equally in this manuscript.

\section{Open Access}

This is an Open Access article that uses a funding model which does not charge readers or their institutions for access and distributed under the terms of the Creative Commons Attribution License (http://creativecommons.org/licenses/by/ 4.0) and the Budapest Open Access Initiative (http://www.budapestopenaccessinitiative.org/rea d), which permit unrestricted use, distribution, and reproduction in any medium, provided the original work is properly credited.

\section{REFERENCES}

1. Okoshi MP, Capalbo RV, Romeiro FG, Okoshi K. Cardiac cachexia: perspectives for prevention and treatment. Arq Bras Cardiol 2017; 108(1): 74-80.
2. Farkas J, von Haehling S, Kalantar-Zadeh K, Morley JE, Anker SD, Lainscak M. Cachexia as a major public health problem: frequent, costly, and deadly. J Cachexia Sarcopenia Muscle 2013; 4(3): 173-178.

3. von Haehling S, Anker SD. Prevalence, incidence and clinical impact of cachexia: facts and numbers-update 2014. J Cachexia Sarcopenia Muscle 2014; 5(4): 261263.

4. Lena A, Ebner N, Coats AJS, Anker MS. Cardiac cachexia: the mandate to increase clinician awareness. Curr Opin Support Palliat Care 2019; 13(4): 298-304.

5. Valentova $M$, Anker SD, von Haehling S. Cardiac cachexia revisited: the role of wasting in heart failure. Heart Fail Clin 2020; 16(1): 61-69.

6. Eash KJ, Greenbaum AM, Gopalan PK, Link DC. CXCR2 and CXCR4 antagonistically regulate neutrophil trafficking from murine bone marrow. J Clin Invest 2010; 120 (7): 2423-2431.

7. Singh S, Varney $M$, Singh RK. Host CXCR2-dependent regulation of melanoma growth, angiogenesis, and experimental lung metastasis. Cancer Res 2009; 69(2): 411-415.

8. Callaway CS, Delitto AE, D'Lugos AC, Patel R, Nosacka $R L$, Delitto $D$, Deyhle MR, Trevino JG, Judge SM, Judge $A R$ et al. IL-8 released from human pancreatic cancer and tumor-associated stromal cells signals through a CXCR2-ERK1/2 axis to induce muscle atrophy. Cancers (Basel) 2019; 11(12): 1863.

9. D'ambrogio A, Nagaoka K, Richter JD. Translational control of cell growth and malignancy by the CPEBs. Nat Rev Cancer 2013; 13(4): 283-290.

10. Xiaoping L, Zhibin $Y$, Wenjuan L, Zeyou W, Gang $X$, Zhaohui L, Ying Z, Minghua W, Guiyuan L. CPEB1, a histone-modified hypomethylated gene, is regulated by miR-101 and involved in cell senescence in glioma. Cell Death Dis 2013; 4(6): e675-e675.

11. Harper SC, Johnson J, Borghetti G, Zhao $H$, Wang $T$, Wallner $M$, Kubo $H$, Feldsott EA, Yang $Y$, Joo $Y$ et al. GDF11 decreases pressure overload-induced hypertrophy, but can cause severe cachexia and premature death. Circ Res 2018; 123(11): 1220-1231.

12. Zimmers TA, Jiang $Y$, Wang $M$, Liang TW, Rupert JE, Au $E D$, Marino FE, Couch ME, Koniaris LG. Exogenous GDF11 induces cardiac and skeletal muscle dysfunction and wasting. Basic Res Cardiol 2017; 112(4): 48.

13. Smith SC, Zhang $X$, Zhang $X$, Gross $P$, Starosta $T$, Mohsin S, Franti M, Gupta $P$, Hayes $D$, Myzithras $M$ et al. GDF11 does not rescue aging-related pathological hypertrophy. Circ Res 2015; 117(11): 926-932.

14. Zhao JX, Zhang HX, Li B, Fan QX, Xue SZ, Zhang M, Ke CQ. Role of MLL3 in regulating cardiac stem cells following cardiac cachexia. Eur Rev Med Pharmacol Sci 2017; 21 (21): 4924-4929.

15. von Haehling $S$, Doehner W, Anker SD. Nutrition, metabolism, and the complex pathophysiology of cachexia in chronic heart failure. Cardiovasc Res 2007; 73(2): 298-309. 
16. Wu $F$, Zhao $Y$, Jiao $T$, Shi $D$, Zhu $X$, Zhang $M$, Shi $M$, Zhou H. CXCR2 is essential for cerebral endothelial activation and leukocyte recruitment during neuroinflammation. J Neuroinflammation 2015; 12(1): 98.
17. Burns DM, Richter JD. CPEB regulation of human cellular senescence, energy metabolism, and p53 mRNA translation. Genes Dev 2008; 22(24): 3449-3460. 